\title{
L'IDENTITÉ MASCULINE ET FÉMININE CHEZ JACQUES MARITAIN FACE AU DOCUMENT DE LA CONGRÉGATION POUR L'ÉDUCATION CATHOLIQUE SUR LE GENRE
}

\author{
LUK Á Š JA N F O ŠU M
}

\section{ABSTRACT \\ The Masculine and Feminine Identity in the Work of Jacques Maritain with Regard to the Document of the Cogregation for Catholic Education on Gender}

The recent document of the Congregation for Catholic Education on the question of gender 'Male and Female He Created Them' emphasises the importance of the sexual difference for the masculine and feminine identity. On the other hand, in his essay 'Faisons-lui une aide semblable à lui' written in 1967, Jacques Maritain insists that this difference is more a difference of the soul than a difference of the body and that it will last also when the bodies are resurrected after death. His concept of the masculine and feminine specificity is based both on biblical and philosophical arguments. Without inclining to feminism, Maritain underlines especially the way in which a woman is different from a man. This study tries to show, how Maritain's ideas about the sub-specificity of man's and woman's soul can be complementary to the anthropology of the above-mentioned document of the Congregation for Catholic Education. Furthermore, it shows also a critical point of view of Maritain's idea of manhood and womanhood with the emphasis on the philosophical terms which are used for the difference between man and woman.

\section{Keywords:}

Jacques Maritain; Philosophical classification of masculinity and femininity; Theological anthropology; Congregation for Catholic Education

DOI: $10.14712 / 23363398.2020 .61$

$\mathrm{L}$ récent document de la Congrégation pour l'éducation catholique (CEC) qui réagit à « diverses formes d’une idéologie, 
généralement appelée "genre"1 " trouve dans la différence sexuelle entre homme et femme un donné fondamental. Ce document considère la différence corporelle comme liée à l'une des inclinations fondamentales de la nature humaine ${ }^{2}$ et qui est ainsi un donné de cette nature. Chez Jacques Maritain, qui a renouvelé de façon importante la vision traditionnelle de la nature humaine, la différence des sexes semble n'être que l'expression d'une différence bien plus profonde entre l'homme et la femme, enracinée d'abord dans l'âme humaine. Dans cet article, nous tâcherons d'exposer plus en détail la position de Maritain sur ce sujet pour voir si elle est compatible avec les avancées du document de la Congrégation. Nous terminerons par une critique de la position maritainienne et nous montrerons pourquoi il faut continuer à chercher une caractéristique philosophique de la masculinité et de la féminité qui soit la plus précise possible.

\section{Le document de la CEC}

Le but du document de la CEC reste d'abord éducatif : comment garder dans l'éducation des jeunes, notamment dans les établissements de l'enseignement catholique, une vision claire et inchangeable de la famille humaine qui se fonde sur la bipolarité, sur la complémentarité et sur la fécondité du couple humain homme-femme, et cela dans un contexte qui relativise profondément ce donné de la nature humaine. Vis-à-vis du « genre » - qui cache non seulement une ou des idéologies, mais aussi une recherche sur l'identité humaine -, le document opte pour la «voie du dialogue - qui écoute, raisonne et propose».

Comment distinguer et articuler le genre et le sexe, la capacité de l'autodétermination libre et les données du corps, la culture et la nature ? Comment éviter « la négation d'un don originaire qui nous précède, qui est constitutif de notre identité personnelle et forme le fondement nécessaire de chacune de nos actions ${ }^{3}$ »? Comment faire promouvoir « une éducation à la citoyenneté active et responsable, où

Congrégation pour l'éducation catholique (CEC), Il les créa homme et femme, 2019, $\mathrm{n}^{\circ} 2$.

"La nature humaine - par un dépassement de tout physicisme ou naturalisme - doit donc être comprise à la lumière de l'unité d'âme et de corps, l'unité de ses inclinations d'ordre spirituel ou biologique et de tous les autres caractères spécifiques nécessaires à la poursuite de sa fin. " (CEC, Il les créa homme et femme, $\mathrm{n}^{\circ}$ 32).

3 Ibid., $\mathrm{n}^{\circ} 9$. 
toutes les expressions légitimes de la personne sont accueillies avec respect $^{4}$ », sans nier " la vérité de l'être ${ }^{5}$ »?

Face à ce défi, le document pose la distinction principale entre le sexe et le genre : "Le sexe définit l'appartenance à une des deux catégories biologiques qui dérivent de la dyade originaire, femme et homme. En revanche, le genre est la manière dont on vit, dans chaque culture, la différence entre les deux sexes ${ }^{6}$. " Dans la formulation de cette détermination de l'identité personnelle de chaque être humain, ce texte magistériel fait écho aux notions traditionnelles de l'union de l'âme et du corps ${ }^{7}$ ou de l'essence ${ }^{8}$, mais son insistance sur la différence sexuelle qui est un donné du corps s'exprime davantage dans une perspective personnaliste qui s'appuie sur le don de soi : « La sexualité, en effet, est une richesse de la personne tout entière - corps, sentiments et âme - et manifeste sa signification intime en la portant au don de soi dans l'amour ${ }^{9}$. " Lintégralité des conséquences de la différence sexuelle montre qu'elle a une racine métaphysique ${ }^{10}$. En plus, dès que l'on commence à réfléchir sur la masculinité et sur la féminité à partir des récits de la Genèse, on réalise que la relation hommefemme se comprend non seulement dans les rapports horizontaux, mais aussi dans sa dimension verticale ${ }^{11}$, qui intègre Dieu-Créateur, celui qui a créé l'homme et la femme à son image et à sa ressemblance.

Rappelons que le document de la CEC saisit la problématique de l'identité homme-femme dans le contexte des recherches contemporaines sur le genre et face aux idéologies avec lesquelles ces recherches peuvent se mélanger. La perspective reste d'abord celle de l'éducation pour laquelle la famille humaine est depuis toujours le premier lieu où elle s'effectue et l'objet d'une la perception juste : les enfants ont besoin d'un père et d'une mère pour grandir à leur tour comme des hommes et des femmes. Avec ce cahier des charges, le document du Magistère utilise aussi un langage philosophique pour caractériser la différence entre la masculinité et la féminité, mais il fait cela dans une certaine pluralité des approches sans préciser vraiment comment dans

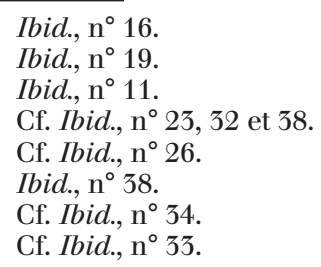


l'essence de l'être humain entre le fait qu'il soit un homme ou qu'elle soit une femme.

\section{Pourquoi chercher chez Maritain?}

Bien que Maritain ait peu écrit sur le thème même de l'identité masculine et féminine, ses propos sont intéressants pour trois raisons au moins. 1) Comme philosophe chrétien, il a renouvelé l'anthropologie aristotélico-thomiste tout en en montrant les points faibles voire dépassés. 2) Parmi les questions anthropologiques, il s'est montré particulièrement attentif à ce qui est relatif et à ce qui est absolu dans l'égalité et la diversité des êtres humains ${ }^{12}$. 3) Grâce à une coopération intellectuelle étroite et fructueuse avec son épouse Raïssa, il a su relever d'une façon à la fois équilibrée et neuve la spécificité et les points forts de l'humanité féminine, surtout sur le plan intellectuel et psychique.

La notion de la nature humaine comme culminant dans la masculinité, si aberrante qu'elle soit, n'a pas fini d'exercer son pouvoir dans notre inconscient, alors que la notion correcte est évidemment celle de la nature humaine comme répartie entre masculinité et féminité, tout en gardant dans l'une et dans l'autre la même valeur et dignité. [...] L'avantage que je reconnais à la femme est en effet chèrement payé par le tribut, beaucoup plus lourd que celui de l'homme, que la nature lui impose en ce qui concerne la propagation de l'espèce. ${ }^{13}$

Dans l'article " Faisons-lui une aide semblable à lui », Maritain se distingue surtout de deux points de vue contraires sur les rapports femme-homme. Le premier point de vue qu'il corrige est celui des Anciens (y compris Thomas d'Aquin) qui regardaient la femme comme un être humain intellectuellement plus faible, dont la fonction principale était la génération de la descendance pour l'espèce humaine. L'autre point de vue, à l'opposé, est le féminisme contemporain présenté par exemple par Yvonne Pellé-Douël ${ }^{14}$. Dans les autres écrits où il

\footnotetext{
12 Jacques Maritain, Principes d'une politique humaniste, in Euvres complètes, Volume VIII (Paris : Éditions universitaires, Fribourg : Éditions Saint-Paul, 1989), 245-277.

13 Jacques Maritain, "Faisons-lui une aide semblable à lui ", in CEuvres complètes, Volume XIII (Paris : Éditions universitaires, Fribourg : Éditions Saint-Paul, 1992), 685. Cet article a d'abord été publié en Nova et Vetera 42 (1967), 241-254.

14 Jacques Maritain, « Faisons-lui une aide semblable à lui », 688.
} 
traite le problème de la masculinité et de la féminité, Maritain conteste les idéologies modernes collectivistes qui portent un regard réducteur sur l'humanité en la considérant uniquement sous sa fonction économique $^{15}$. Il propose une réflexion complexe qui se forme à partir des récits fondateurs du livre de la Genèse et qui se lie avec la philosophie de l'âme humaine donne la base des arguments contre ces différentes sortes d'erreurs.

\section{Méditation biblique, philosophique et théologique}

Le fait de s'appuyer sur les premières pages de l'Écriture Sainte pourrait paraître banal, voire simpliste. Or, « la tradition judéo-chrétienne - justement dans l'ouverture grandiose du récit de la Genèse donne une formulation décisive des notions d'humanité, de personnalité, d'identité, et elle le fait de manière égale pour l'homme ou pour la femme ${ }^{16}$ ». De plus, " quelque chose du trésor anthropologique et théologique du récit de la Genèse et du Nouveau Testament est resté enfoui dans le champ de l'histoire $»{ }^{17}$

Larticle « Faisons-lui une aide semblable à lui » est la contribution principale de Maritain aux questions tant discutées aujourd'hui de l'identité sexuelle et de son fondement. Le titre laisse deviner que les récits bibliques de la création de l'être humain seront plus qu'un simple point de départ : ils constituent le fondement perpétuel de l'égalité et de la diversité de l'homme et de la femme. Maritain approfondit cette question par des éléments philosophiques et eschatologiques. Cela donne une vision d'ensemble de l'homme et de la femme dans leur vocation commune et partagée qui a comme but principal d'être à l'image et à la ressemblance de Dieu. Cette conception se déploie en cinq thèses que nous allons maintenant présenter : les trois premières puisent directement dans les chapitres $1-3$ de la Genèse. La quatrième est une thèse philosophique sur le rapport de l'âme au corps humain. Enfin, la cinquième thèse regarde ce que l'humanité a dans

15 Cf. Jacques Maritain, "Humanisme intégral ", in CEuvres complètes, Volume VI, 1984, 511-515.

16 Hanna-Barbara Gerl-Falkovitz, "La personnalité chez l'homme et la femme ", in François-Xavier Putallaz, Bernard N. Schumacher (éd.), L'humain et la personne (Paris : Cerf, 2008), 384.

17 Ibid, 383. 
sa sub-spécificité masculine et féminine à voir avec la finalité propre de l'être humain.

Dans leur vocation d'êtres créés à l'image et à la ressemblance de Dieu, l'homme et la femme sont égaux. Ce n'est qu'ensemble qu'ils sont porteurs de cette vocation. En Gn 1, 27, l'expression " "homme et femme il les créa", est synonyme de l'Homme (avec une majuscule), et le constitue dans sa plénitude ontologique ». ${ }^{18}$

Dans Gn 2, 21-23 il est présenté de quelle façon la femme se distingue de l'homme depuis le commencement. "Ève n'a pas été tirée directement comme Adam, du limon de la terre, mais d'une matière plus élaborée et plus raffinée, d'une chair déjà vivante et humaine, ce qui veut dire qu'elle a reçu en partage les qualités les plus délicates et du plus haut prix pour la race humaine ${ }^{19}$

La domination de l'homme sur la femme n'est pas le résultat de cette manière différente d'être créée. Il s'agit, d'après Gn 3, 16, de la conséquence du péché originel et cela est imposé aux êtres humains « à titre de châtiment.$^{20}$

L'âme spirituelle n'est pas "individuée par la matière ", mais " en ordre à la matière ». ${ }^{21}$ Par conséquent, la sub-spécificité masculine est davantage une propriété de l'âme que celle du corps.

La différence de l'homme et de la femme demeure, qu'ils accomplissent ou non leur vie conjugale et l'œuvre de la reproduction. Elle n'est pas seulement de l'ordre temporel, elle sera présente aussi dans le monde des ressuscités.

Si on regarde maintenant les trois premières thèses bibliques ensemble, il faut d'abord souligner que de tels phénomènes, présents tout au long de l'histoire - tels que la domination de l'homme sur la femme, son contraire que l'on trouve de nos jours dans le féminisme (ou déjà avec le matriarcat), la rivalité entre la femme et l'homme en général ou encore la tendance à accaparer les dons propres à l'autre tout cela appartient aux effets du péché des origines, et non pas à la différence entre l'homme et la femme conçue par la Sagesse créatrice.

\footnotetext{
Jacques Maritain, « Faisons-lui une aide semblable à lui », 682.

Ibid, 683.

20 Ibid., 695. Dans ST I, q. 92, a. 1, ad 2, saint Thomas distingue deux types de sujétion de la femme à l'homme : une, qui est servile et comme une conséquence du péché, et l'autre, primordiale, qui vient déjà de l'état d'innocence : la femme est naturellement plus faible et moins digne que l'homme. Selon le livre de la Genèse, l'homme est le principe de la femme.

Ibid, 692 .
} 
Dieu a voulu que l'homme et la femme s'aident mutuellement et réciproquement dans leur chemin vers la plénitude de la vie à l'image de Dieu. Cette complémentarité se manifeste dans les qualités psychiques plus encore que dans les différences corporelles : si la force d'âme masculine se retrouve dans la puissance conceptuelle, dans la capacité du gouvernement et dans l'exercice d'une autorité juste, l'âme féminine est quant à elle dotée de finesse et de capacités intuitives. Le fait d'être créée à partir de la matière plus fine, " chair de la chair ", a donc des conséquences non seulement pour l'apparence du corps féminin, mais aussi pour le fonctionnement de son âme humaine.

La psychologie philosophique aristotélico-thomiste permet d'ordonner et d'articuler les propriétés psychiques et les propriétés corporelles : l'âme, qui est la forme et l'acte (entelechia) du corps, est à l'origine de la spécificité de l'être humain - qu'il soit homme ou femme. Les propriétés masculines ou féminines sont ainsi fondées davantage dans l'âme que dans le corps. Ainsi, les différences sexuelles ne sont qu'une manifestation d'une différence plus profonde qui est inscrite dans la sub-spécificité de l'âme spirituelle : celle-ci, à la différence de l'âme animale, ne s'individualise pas à partir de la matière (ex qua), mais dans la matière (in $q u a^{22}$ ) ou comme le dit Maritain : "en ordre à la matière et à un corps déterminé, mâle ou femelle ${ }^{23}$ ». La différence sexuelle qui est liée aux fonctions temporelles du corps n'est pas donc ce qui est le plus essentiel à la sub-spécificité masculine ou féminine, car ces dernières sont d'abord les propriétés perpétuelles de l'âme qui demeurent les mêmes une fois que les fonctions unitives et reproductives des corps cessent d'être actuelles.Il s'agit selon Maritain d'une propriété modale essentielle. L'essence humaine reste une seule pour les hommes et pour les femmes. Elle est néanmoins dans chaque personne individualisée soit dans le mode masculin soit dans le mode féminin, ${ }^{24}$ ce qui a des implications pour toute la personne humaine, son corps et son âme. P.-M. Margelidon résume ainsi la conception maritainienne de la masculinité et de la féminité :

22 «Individuation », in Philippe-Marie Margelidon, Yves Floucat, Dictionnaire de philosophie et de théologie thomistes, Bibliothèque de la Revue Thomiste (Paris : Parole et silence, 2016), 217-218.

25 Jacques Maritain, «Faisons-lui une aide semblable à lui », 692.

24 Maritain exprime cette bipolarité aussi avec un parallèle chimique : « l'âme d'un homme est dès sa création dextroversement individualisée et celle d'une femme lévoversement individualisée. " (Ibid., 692). 
L'espèce humaine est constituée et partagée en deux types subspécifiques, le masculin et le féminin, qui ne sont pas purement fonctionnels mais qui correspondent à des différences ontologiques. Ces deux types subspécifiques sont d'ordre modal-essentiel, c'est dire qu'ils correspondent aux deux modes de l'essence humaine. Il y a donc une différence " de nature » entre féminité et masculinité selon que les caractéristiques de l'un et de l'autre affectent la nature humaine tout entière. Celles-ci relèvent plus profondément de l'ordre psychique et spirituel que de l'ordre corporel..$^{25}$

Ce donné philosophique qui met en avant les propriétés de l'âme humaine avant celle du corps s'éclaire encore plus dans la thèse que nous appelons eschatologique. Maritain insiste : dès les origines de l'homme, qu'il s'agisse de la création elle-même ou de ce qui suit après le premier péché, la vie sexuelle et la reproduction des hommes n'est qu'une étape en vue d' " une vie spirituelle de plus en plus élevée » dont le but est d'« entrer finalement dans le royaume de Dieu et de la vision béatifique. $»^{26}$

\section{Les points saillants de la position de Maritain}

Maritain veut surtout éviter que l'on considère « le sexe et la fonction génitale comme le fondement premier d'où dérivent et par où s'expliquent tous les autres caractères différentiels qui distinguent l'êtrehomme et l'être-femme ${ }^{27}$ ». Pour cela, il s'appuie sur l'anthropologie philosophique fondée sur l'individuation de l'âme humaine non à partir du corps mais en ordre à un corps et sur les distinctions fondamentales entre l'homme et la femme révélées dans la Genèse. Sans verser dans l'extrême opposé à celui des siècles précédents, Maritain souligne surtout ce qui est propre à la femme comme à celle qui a été créée à partir de l'homme pour être son vis-à-vis.

Dans sa méditation « Faisons-lui une aide semblable à lui », Maritain insiste sur les propriétés sub-spécifiques de l'homme et de la femme

25 Philippe-Marie Margelidon, Les Fins dernières. De la résurrection du Christ à la résurrection des morts (Paris : Artège - Lethielleux, 2016), 85 cité par Isolde Cambournac, La masculinité et la féminité à la lumière de l'anthropologie de Thomas d'Aquin, Thèse du doctorat soutenue dans la faculté de théologie de l'Université de Fribourg, Fribourg, 2018, http://doc.rero.ch/record/324533, 259.

27 Ibid, 694-695. 
qui leurs sont données dès l'instant de leur création (on pourrait aussi dire : dès leur établissement dans l'humanité). Ces propriétés sub-spécifiques sont d'abord ancrées dans l'âme d'un être humain avant d'être exprimées dans son corps, car la spécificité sexuelle n'a qu'une fonction temporelle et elle est subordonnée à la finalité spirituelle. Lidentité masculine et féminine est plus ancrée dans l'âme que dans le corps, parce que l'âme est le principe à partir duquel le corps se forme.

Dans son combat contre la réduction des différences aux signes sexuels, Maritain semble aller trop loin en parlant du sexe comme du parasite de la personne:

Le sexe ne fonde que la différence animale, si importante qu'elle soit, et si immédiatement évidente ; il ne fonde pas les différences proprement humaines entre l'homme et la femme. Le sexe et la fonction de reproduction sont en effet pour l'espèce et pour la perpétuation de l'espèce, et en ce sens ce sont des parasites de la personne. ${ }^{28}$

C'est là où le propos de Maritain se distingue le plus de celui du document récent de la Congrégation pour l'éducation catholique qui, au contraire, pose la différence des sexes comme un des fondements de l'identité personnelle. Pour autant, dans les deux cas les auteurs s'efforcent d'intégrer l'identité sexuelle dans la personne humaine. Il faut donc voir maintenant quelles pourraient être les différences significatives entre Maritain et le Magistère contemporain dans leur compréhension de la personne humaine.

\section{La différence de la perspective de Maritain de celle du document de la Congrétation}

Si les deux perspectives ici mentionnées visent l'être humain comme une personne, la différence entre elles se dessine à partir de l'usage du concept « âme ». Après avoir établi que « du sexe, en effet, la personne humaine reçoit les caractères qui, sur le plan biologique, psychologique et spirituel, la font homme et femme, conditionnant par-là grandement son acheminement vers la maturité et son insertion dans la société », le document de la Congrégation élargit la perspective en affirmant qu'« une telle diversité, connexe à la complémentarité des

28 Jacques Maritain, « Faisons-lui une aide semblable à lui », 686. 
deux sexes, répond pleinement au dessein de Dieu selon la vocation à laquelle chacun est appelé ${ }^{29}{ }^{29}$

Maritain de son côté intègre également la sexualité dans la personne humaine. Cette intégration s'effectue néanmoins à partir de l'individuation de l'âme ordonnée au corps et elle subordonne le pôle charnel au pôle spirituel :

La personne humaine étant celle d'un animal doué de raison, et l'âme humaine étant individualisée en ordre à un corps déterminé, le sexe et la fonction de génération, avec tout ce qui leur est lié dans le fonctionnement interne de la vie organique - surtout chez la femme, qu'il s'agisse de l'espèce de complicité qui s'établit ainsi entre elle et la nature, ou qu'il s'agisse surtout de la gestation, où l'enfant est dans le sein maternel comme dans un nid naturellement sacré - créent dans l'animalité humaine une sorte de consonance ou de correspondance harmonique avec ce qui, au pôle spirituel, et du côté de l'âme et de ses qualités particulières, constitue la personne humaine dans l'être-homme ou l'être-femme. ${ }^{30}$

Lapproche du document de la Congrégation recherche la signification du don de soi dans l'altérité je-tu et dont le corps est l'instrument $^{31}$, tandis que Maritain met davantage l'accent sur l'ordonnancement entre l'âme et le corps, le temporel et le spirituel. Le document de la Congrégation insiste sur la totalité et l'instantanéité du don du soi qu'on ne peut faire autrement qu'en accord avec son être tel qu'il est formé dès le sein maternel. Pour Maritain, ce qui est crucial, c'est la subordination du temporel au spirituel, celle de la manifestation charnelle à la sub-spécificité de l'essence. Le document de la Congrégation lutte contre le dualisme qui sépare « un corps réduit à la matière et une volonté qui devient absolue » et pour lequel « le gender (genre)

29 CEC, Il les créa homme et femme, $\mathrm{n}^{\circ}$ 4. Et plus loin : « À la lumière d'une écologie pleinement humaine et intégrale, la femme et l'homme reconnaissent la signification de la sexualité et de la génitalité dans cette intentionnalité relationnelle et communicative intrinsèque qui traverse leur corporéité et les renvoie mutuellement l'un vers l'autre. » (Ibid., $\left.\mathrm{n}^{\circ} 35\right)$.

30 Jacques Maritain, « Faisons-lui une aide semblable à lui », 694.

31 «Puisque le corps est le signe du don, il en est aussi l'instrument. En effet, il est instrument du don réciproque des époux, de la communion des personnes. " (Thibaud Collin, La Théologie du corps de Jean-Paul II : quelle application à la sexualité contemporaine ? in Denis Borel et Jean-Gabriel Goupil de Bouillé (éd.), La Personne en débat, Bibliothèque de la Revue Thomiste (Paris : Parole et silence, 2008), 239). 
finit par être plus important que le sex (sexe) ${ }^{32}$. " Maritain formule une anthropologie équilibrée qui évite à la fois le dualisme et le monisme, et qui accorde moins d'importance aux seules différences corporelles entre la femme et l'homme que le document qui réagit face au gender.

Enfin, la différence subtile entre les titres des deux textes que nous comparons principalement ici est déjà significative. D'un côté « Il les créa homme et femme » (Gn 1,27) dit que l'être humain est dès le début soit homme, soit femme, et que l'un et l'autre ont été créés pour être ensemble : la masculinité et la féminité sont créées dès le début par Dieu dans la complémentarité. De l'autre côté, "Faisons-lui un aide semblable à lui » $(\mathrm{Gn} 2,18)$ montre la différence de la manière dont la femme a été créée et qu'il y a un chemin - Maritain dira " un progrès » que l'homme et la femme ont à accomplir.

Ainsi, la contribution de Maritain aux débats sur le "gender » consiste dans son insistance sur la perpétuité de la différence hommefemme qui est enracinée dans l'âme humaine. La bipolarité sexuelle avec les fonctions corporelles qui lui correspondent se trouve ainsi non relativisée, mais subordonnée à la vocation d'être à l'image et à la ressemblance de Dieu qui s'étend au-delà de la vie conjugale et familiale. Maritain ouvre d'un côté la porte aux avis qui disent que les signes corporels ne sont pas si importants pour l'identité masculine ou féminine, de l'autre côté il absolutise la différence homme-femme comme celle qui est instituée par Dieu depuis la création de l'être humain et qui demeure jusqu'au monde des ressuscités et qui n'est donc pas une option que l'on pourrait manipuler. Maritain prend au sérieux l'enseignement du Magistère selon lequel l'âme est la forme du corps $;^{33}$ sur ce point, il souligne l'aspect de l'ordre qui est présent depuis le commencement jusqu'à l'éternité dans l'être humain.

\section{La critique de la vision maritainienne de la sub-spécifité masculine et féminine}

Après avoir saisi les différences principales entre les accents philosophiques, bibliques et eschatologiques de Maritain d'un côté, et l'approche de la phénoménologie du don du document récent de la Congrégation pour l'éducation catholique de l'autre côté, nous pouvons voir maintenant

32 CEC, Il les créa homme et femme, $\mathrm{n}^{\circ} 20$.

33 Catéchisme de l'Eglise catholique, , $\mathrm{n}^{\circ} 365$. 
une critique de la position maritainienne qui procède davantage de la même école. Dans sa thèse doctorale sur la masculinité et la féminité, Isolde Cambournac revient à plusieurs reprises sur le point de vue de Maritain. Son argumentation contre la « sub-spécificité » masculine et féminine, promue par Maritain, est avant tout philosophique, fondée sur la méthode aristotélicienne de saint Thomas d'Aquin. Qu'est-ce la masculinité selon l'analyse catégorique de l'être ? Selon Cambournac, la masculinité et la féminité ne sont ni un accident inséparable comme disait Thomas d'Aquin, ni un type subspécifique humain, comme disait Maritain. La précision consiste dans le discernement sur les accidents prédicables $^{34}$ et sur les accidents prédicamentaux (ou prédicaments). ${ }^{35}$

La masculinité est la puissance active d'engendrer, et la féminité est la puissance passive d'engendrer. Ces deux puissances sont complémentaires et nécessaires pour la génération. Cela répondait à notre question sur les prédicables : si la masculinité et la féminité sont des puissances d'engendrer, alors ce sont des propres. Ce ne sont ni des accidents inséparables comme le pensait Thomas, ni des types subspécifiques comme le pensait Jacques Maritain. Le propre se situe d'une certaine manière entre l'accident inséparable et le type subspécifique. Comme elles sont des propres, la masculinité et la féminité découlent des principes de l'espèce. Cependant, ce sont les seuls propres qui ne sont pas présents chez tous les individus de l'espèce humaine. La moitié des individus a le propre de la masculinité, et l'autre moitié le propre de la féminité : ce sont donc des propres disjonctifs. ${ }^{36}$

Ces propres disjonctifs, masculinité et féminité, ajoute Cambournac, ont leur principe dans l'essence de l'âme et leur sujet dans les organes de la génération ${ }^{37}$. Maritain avait donc, selon Cambournac, raison d'avoir fondé les différences entre l'homme et la femme dans

34 «Les prédicables sont des classes plus ou moins universelles. Ils permettent à l'intellect de situer des attributs par rapport à un sujet. En associant un attribut à une classe et donc à un prédicable, l'intellect peut savoir si cet attribut est plus ou moins universel par rapport au sujet. " (Isolde Cambournac, La masculinité et la féminité à la lumière de l'anthropologie de Thomas d'Aquin, Thèse de doctorat: Université de Fribourg, 2018, 254.).

35 « On distingue dix prédicaments (ou catégories) : la substance et les neuf accidents (la quantité, la qualité, la relation, le temps, le lieu, la possession, la situation, l'action et la passion). Contrairement aux prédicables où l'on distingue le propre, il n'y a pas d'intermédiaire dans les prédicaments entre la substance et les accidents. » (Ibid., 273) La puissance de la génération est une qualité.

36 Ibid., 355.

37 Ibid., 404. 
l'essence de l'âme humaine, mais sa catégorie de la «sub-spécificité » ne semble pas être très précise : il est plus juste de classer la masculinité et la féminité entre les propres disjonctifs.

Le point le plus problématique de Maritain est donc ce terme de la sub-spécificité, parce qu'il met en question la définition de l'espèce humaine. Le fait de classer la masculinité et la féminité parmi les accidents n'implique point une réduction au temporel et négligeable. Qu'il soit un accident inséparable ou un propre disjonctif, dans les deux cas il s'agit d'un attribut durable avec des conséquences pour l'individuation. Par contre, l'usage du terme de « sub-spécificité » met sérieusement en question la différence spécifique : chez l'être humain, c'est la rationalité, aussi bien chez l'homme que chez la femme. Même si la rationalité peut avoir des tonalités différentes dont certaines ${ }^{38}$ que l'on observe plus souvent chez les femmes que chez les hommes et vice-versa, elle reste en soi une propriété, une spécificité humaine qui nous tous, hommes et femmes, distinguent des autres animaux.

\section{Ce que le texte de Maritain dit et ce qu'il ne dit pas}

Si nous revenons à ce donné fondamental qui distingue l'homme de la femme et qui est cherché par le document de la CEC, et établi aussi à sa façon, par Maritain, le philosophe français ne relativise point «le pôle charnel » de la différence sexuelle : il le subordonne au pôle spirituel. A la différence du document de la CEC, Maritain ne développe pas une réflexion sur la sexualité humaine. En élevant la masculinité et la féminité au niveau de la sub-spécificité, il ne relativise pas la différence sexuelle (et cela est vrai malgré l'expression un peu malheureuse : «parasite de la personne »), mais il l'enracine dans la propriété éternelle de l'âme humaine.

Maritain soutient sa lecture de Gn 1, 26 et 2,18-24 avec la liste des propriétés de l'âme masculine et féminine, mais il ne les formalise pas comme des exclusivités qui impliqueraient qu'un homme ne pourrait pas naturellement avoir certaines caractéristiques de l'intelligence plutôt féminine et vice-versa sans que cela signifie une déviation.

A la différence d'Isolde Cambournac, Maritain ne dit pas où est le sujet de la masculinité et de la féminité dans l'être humain. Il semble

38 Maritain attire particulièrement notre attention sur les propriétés typiquement masculines et féminines de l'esprit. 
que selon lui, cette propriété de l'âme pénètre absolument tout le corps : l'être humain vit et fonctionne différemment dès le moment de sa création propre à l'image et à la ressemblance de Dieu, qui est soit masculine, soit féminine.

Maritain de son côté part dès le début du donné biblique : l'homme et la femme sont créés à l'image et à la ressemblance de Dieu. La capacité de la génération qui est transitoire dans la longueur de leurs existences éternelles ne peut donc pas être prise comme une distinction la plus fondamentale, comme le propre disjonctif, car elle n'est, selon Maritain, qu'une manifestation de la différence qui se trouve plus profondément enracinée dans l'âme.

On pourrait certainement reprocher à Maritain la négligence des donnés biologiques, si importants, dans les piliers de sa réflexion. Or, l'égalité de l'homme et de la femme que Maritain affirme, présuppose les connaissances de la biologie moderne et dépassent l'idée aristotélicienne, reprise par Thomas d'Aquin que la femme est dès l'embryogenèse " un homme manqué ». ${ }^{39}$ Même si les connaissances biologiques que nous avons aujourd'hui sur les différences sexuelles et sur le rôle de l'homme et de la femme dans la génération changent considérablement le point de vue porté notamment sur la femme par rapport à la vision aristotélico-thomiste, Maritain part toujours de l'idée principale que les différences sexuelles et la fonction de la génération ne sont pas déterminantes et fondatrices dans la bipolarité homme-femme. Lévènement, le trait fondateur que le philosophe français traduit en «type sub-spécifique ", c'est le mode d'être créé à l'image et à la ressemblance de Dieu. Maritain prolonge dans la lumière des Ecritures cette différence jusqu'à l'éternité.

\section{Conclusion}

Les deux propos sur le fondement de la différence entre homme et femme que nous avons abordés dans cette étude se distinguent sans doute d'abord par ce à quoi ils réagissent. Le document récent de la Congrégation pour l'éducation catholique veut surtout préserver la détermination naturelle du sexe contre la fluidité et l'ambiguïté du «gender» qui se greffe sur un dualisme anthropologique. Maritain a de

\footnotetext{
39 «Femina dicitur mas occasionatus, quia est praeter intentionem naturae particularis, non autem praeter intentionem naturae universalis. » (STI, q. 99, a. 2, ad 1).
} 
son côté réagi contre les tendances trop matérialistes qui réduisaient les différences entre homme et femme aux organes corporels et à leurs fonctions. Il s'efforçait d'éviter en même temps les deux extrêmes que sont l'extrême "traditionnel » de la préférence accordée à l'homme et l'extrême moderne du féminisme. Le philosophe français montre la force d'une réflexion qui articule données bibliques et instruments philosophiques. Il a su relever les points forts de l'humanité féminine et a rappelé en quoi consiste la finalité de chaque être humain, homme ou femme, clerc, religieux ou laïc. Il n'a pas développé de signification de la sexualité humaine, ni son intégration dans la personne humaine.

La critique de la position maritainienne a montré qu'un autre classement philosophique de la masculinité et de la féminité que la sub-spécifité de l'essence s'avère plus juste : la puissance de la génération, dont le principe est toujours l'âme humaine et le sujet les organes engagés dans la génération masculine et féminine, est un propre disjonctif. La formulation philosophique de la différence homme-femme du document de la CEC reste ouverte à la fois à un approfondissement du type "disjonctif propre ${ }^{40}$ », car il utilise l'expression " différence sexuelle $^{41}$ », ainsi qu'au terme de la sub-spécificité, car il utilise aussi l'expression «formes complémentaires ${ }^{42}$ » ou " les deux modalités selon lesquelles s'exprime et se réalise la réalité ontologique de la personne humaine $\mathrm{e}^{43}$ ». Cela montre que la classification philosophique plus précise de la masculinité et la féminité reste encore une question ouverte, même si on est loin de la relativiser et de la faire passer aux influences culturelles et aux choix individuels.

Catholic Theological Faculty, Charles University

Thákurova 3

16000 Praha 6

Czech Republic

E-mail:fosumj@ktf.cuni.cz

\footnotetext{
Cf. $n^{\circ} 34$.

Cf. $n^{\circ} 11,21,25$ et surtout 26 .

Cf. Ibid. $\mathrm{n}^{\circ} 34$.

Cf. Ibid.
} 